\title{
Mesoscopic description of the equal-load-sharing fiber bundle model
}

\author{
Martin Hendrick, ${ }^{*}$ Srutarshi Pradhan,${ }^{\dagger}$ and Alex Hansen ${ }^{\ddagger}$ \\ PoreLab, Department of Physics, Norwegian University of Science and Technology, 7491 Trondheim, Norway
}

(Received 23 February 2018; published 12 September 2018)

\begin{abstract}
One aim of the equal-load-sharing fiber bundle model is to describe the critical behavior of failure events. One way of accomplishing this is through a discrete recursive dynamics. We introduce a continuous mesoscopic equation catching the critical behavior found through recursive dynamics. It allows us to link the model with the unifying framework of absorbing phase transitions traditionally used in the study of nonequilibrium phase transitions. Moreover, it highlights the analogy between equal-load-sharing and spinodal nucleation. Consequently, this work is a step toward the quest of a field theory for fiber bundle models.
\end{abstract}

DOI: 10.1103/PhysRevE.98.032117

\section{INTRODUCTION}

While equilibrium phase transitions are today well understood, a general framework to study the nonequilibrium counterpart is still lacking. Recently, major efforts have been invested into identifying the universality classes related to nonequilibrium phase transitions. The theory of absorbing phase transitions (APTs) is emerging as a unifying framework (or, more generally, field theory applied to nonequilibrium scaling behavior) [1,2]. An absorbing phase transition occurs when a system leaves an active state and enters an absorbing state from which the system cannot escape by itself.

The APT formalism improved the understanding of the universal behavior of a great variety of models such as epidemic and population dynamics [3], sandpiles [4], interfaces in random media [5], and reaction-diffusion systems [2]. The most prominent and generic nonequilibrium universality class is directed percolation, which is believed to describe the phase transition toward a unique absorbing state of systems that is not characterized by any special symmetry (except, effectively, the rapidity reversal symmetry) or conservation law. This is known as the Janssen-Grassberger directed percolation conjecture $[6,7]$.

Fiber bundle models (FBMs) describe rupture phenomena as irreversible fiber breaking processes through discrete breaking rules $[8,9]$. In their simplest form, they consist of two stiff parallel clamps with fibers between them. All the fibers have the same elastic constant. However, the maximum force each fiber can sustain before it fails irreversibly is set by a threshold drawn for each fiber from a probability distribution. Due to irreversibility, detailed balance does not hold. Therefore, fiber bundle models are nonequilibrium systems. We focus here

\footnotetext{
*martin.hendrick@ntnu.no

${ }^{\dagger}$ srutarshi.pradhan@ntnu.no

‡alex.hansen@ntnu.no
}

Published by the American Physical Society under the terms of the Creative Commons Attribution 4.0 International license. Further distribution of this work must maintain attribution to the author(s) and the published article's title, journal citation, and DOI. on the dynamical description of the equal-load-sharing (ELS) model, which is the mean-field (MF) limit of the fiber bundle models [10]. This is the model we sketched earlier in this paragraph. The equal-load-sharing fiber bundle model may be described through a discrete recursion relation (recursion dynamics) [11]. It was shown that ELS undergoes a phase transition.

The aim of this work is to derive a mesoscopic equation encapsulating the ELS critical dynamics. We show the close formal connection, in the limit of vanishing external field, between ELS critical behavior and an APT process: the compact directed percolation (CDP) model. Next we show that the ELS mesoscopic equation can be derived as a purely relaxational model depending on a Hamiltonian describing the ELS stationary behavior. Then, based on a symmetry argument, we highlight the origin of the analogy between the FBM and spinodal nucleation [12,13].

In Sec. II we review the critical properties of the fiber bundle model using recursive dynamics. In Sec. III we provide a mesoscopic description of ELS. We numerically compare ELS and the mesoscopic equation in Sec. IV. We proceed in Sec. V to show the phenomenological and formal similarities between ELS and CDP in an external field. Section VI demonstrates how the ELS fiber bundle model may be described through an overdamped Langevin equation. Then, in Sec. VII we explicitly link the FBM and spinodal nucleation. In Sec. VIII we summarize and discuss our work.

\section{RECURSIVE DYNAMICS OF THE EQUAL-LOAD-SHARING FIBER BUNDLE MODEL}

The ELS fiber bundle model describes the breaking process of $N$ initially intact fibers subject to a homogeneous external field $\sigma$, the initial load per fiber. A fiber $j$ is characterized by a strength $\tau_{j}$, which is a threshold value sampled from a probability distribution $p$. We denote by $n(t)$ the number density (number of surviving fibers divided by $N$ ) of intact fibers at time $t ; k(t)=1-n(t)$ is the density of broken fibers (the damage) with $n(0)=1$. The dynamics of the system under load is defined as follows. At discrete time $t>0$, all fibers $j$ 
such that

$$
\tau_{j}<\frac{\sigma}{n(t-1)}
$$

break irreversibly. Then the number of intact fibers is updated and the process continues until the system reaches a stationary configuration. We note that, by definition, the model is infinitely dimensional, i.e., space plays no role. Local load redistribution introduces a spatial effect in the fiber bundle model and is studied, for example, in the local-load-sharing fiber bundle model where the nearest neighbors of the failed fibers absorb the load they were carrying at failure $[8,9]$.

The control parameter of ELS is $\sigma$. As we will see below, ELS exhibits critical behavior close to the critical point $\sigma=\sigma_{c}$. The exponents characterizing the system in the vicinity of the critical point do not depend on the choice of the threshold probability (see $[8,9,12])$. In the following we will work with the uniform threshold distribution for simplicity.

Formally, the system dynamic is described by a recursive relation $[8,9]$. The density of broken fiber $k(t)$ is given by the threshold cumulative distribution $P(\tau)=\int_{0}^{\tau} p\left(\tau^{\prime}\right) d \tau^{\prime}$,

$$
k(t)=P\left(\frac{\sigma}{n(t)}\right)=\int_{0}^{\sigma / n(t)} p(x) d x .
$$

Thus,

$$
n(t+1)=1-P\left(\frac{\sigma}{n(t)}\right)=1-\frac{\sigma}{n(t)}
$$

since $P(\tau)=\tau$ for the uniform distribution on the unit interval. The breaking process occurs until the system reaches a stationary configuration

$$
n_{*}=1-\frac{\sigma}{n_{*}},
$$

with $n_{*}$ the stationary solution of Eq. (3). Therefore, the equation of state for the stable system is

$$
n_{*}^{2}-n_{*}+\sigma=0 .
$$

Defining the system order parameter as $\eta=n_{*}-1 / 2[8,9]$, we observe that

$$
\eta \sim\left(\sigma_{c}-\sigma\right)^{\beta},
$$

with $\sigma_{c}=1 / 4$ and $\beta=1 / 2$ as the order parameter exponent. Thus, to keep $\eta$ real, we study the system for the load $\sigma \leqslant \sigma_{c}$.

At the critical point, i.e., at $\sigma=\sigma_{c}$ and for $t \rightarrow \infty$, given Eq. (3), we have

$$
\eta \sim t^{-\alpha},
$$

with $\alpha=1$. This characterizes the critical slowing down of the fiber bundle model.

Other standard universal exponents are found in the same way. The susceptibility is

$$
\chi=\left|\frac{\partial \eta_{*}}{\partial \sigma}\right| \sim\left(\sigma_{c}-\sigma\right)^{\beta-1}=\left(\sigma_{c}-\sigma\right)^{-\gamma},
$$

with $\gamma=1 / 2$ the susceptibility exponent. Note that, since the model is governed by only one physical parameter, which is the external load $\sigma$, the susceptibility exponent depends directly on
TABLE I. Mean-field exponents of the fiber bundle model (ELS) using standard notation.

\begin{tabular}{ll}
\hline \hline Notation & ELS \\
\hline$\beta$ & $1 / 2$ \\
$\gamma$ & $1 / 2$ \\
$v_{\|}$ & $1 / 2$ \\
$\alpha$ & 1 \\
\hline \hline
\end{tabular}

the order parameter. The relaxation time $\xi_{\|}$toward a stationary solution follows [8]

$$
\xi_{\|} \sim\left(\sigma_{c}-\sigma\right)^{-v_{\|}},
$$

with $v_{\|}=1 / 2$ being the time correlation length exponent. We summarize the ELS universal exponents in Table I.

The continuous limit of Eq. (3) can be readily obtained and is [8]

$$
\frac{\partial n}{\partial t}=-\frac{n^{2}-n+\sigma}{n} .
$$

The presence of the density in the denominator of this equation makes it hardly amenable for a standard field theory treatment. In the following we introduce an alternative mesoscopic equation.

\section{MESOSCOPIC ELS EQUATION}

We show how to simplify, keeping the same critical behavior, the ELS continuous equation (10). Introducing the order parameter $\eta=n-1 / 2$ in Eq. (10), we have

$$
\frac{\partial \eta}{\partial t}=-\frac{\eta^{2}-J}{\eta+1 / 2}
$$

where $J=\sigma_{c}-\sigma=1 / 4-\sigma$. In the double limit $\eta \ll 1 / 2$ and $J \rightarrow 0$, we can write

$$
\frac{\partial \eta}{\partial t} \approx 2\left(-\eta^{2}+J\right)
$$

We observe, in the limit of $t \rightarrow \infty$, that we recover exactly stationary behavior of the ELS model. We further simplify the equation by absorbing the factor 2 in the time parameter,

$$
\frac{\partial \eta}{\partial t}=-\eta^{2}+J
$$

This generalized equation encapsulates the mesoscopic behavior of the ELS model.

Indeed, for example, the order parameter exponent is given by

$$
\eta_{*} \sim J^{\beta}
$$

with $\eta_{*}$ the stationary solution and $\beta=1 / 2$. At criticality, i.e., for $J=0$, we have

$$
\eta_{c}(t) \sim t^{-\alpha}
$$

where $\alpha=1$. The susceptibility and time correlation length can also be computed easily (see, for example, [14]). It appears that Eq. (13) reproduces the universal exponents of the ELS model. The scaling forms of ELS and the mesoscopic equation (13) are studied in the next section. 
Equation (13) (among other microscopic interpretations) coincides with the mean-field rate behavior of a coagulation with input $(\mathrm{CI})$ reaction-diffusion process, where $\eta$ is the particle density. More precisely, CI describes particles $A$ diffusing on a lattice that coagulate when they meet $(A+A \rightarrow$ $A$ ) with a source term $(\emptyset \rightarrow A)$ acting at rate $J$. This system is characterized by an upper critical dimension $d_{c}^{u}=2$. It was extensively studied by Droz and co-workers (see $[15,16]$ ). The mean-field rate equation (13) holds for CI above $d=2$. This formal similarity between $\mathrm{CI}$ and the FBM invites us to formulate the latter using reaction-diffusion or epidemic propagation phenomenon terminology (see Sec. V).

\section{DATA COLLAPSE}

In the preceding section we showed that the mesoscopic description is obtained as the limit $t \rightarrow \infty$ and/or $J \rightarrow 0$ of the ELS dynamics. By definition, two systems belong to the same universality class if they have the same critical exponents and, near the critical point, if their scaling functions are identical. With this aim, we numerically solve and compare Eqs. (10) and (13). We note that while the exact solution of Eq. (13) is easy to obtain, a numerical approach is needed for the ELS one. Hence, we carry out the data collapse for different solutions close to the critical point.

We note that $\eta_{\mathrm{ELS}}(t, J)=n_{\mathrm{ELS}}(t, J)-1 / 2$ is the shifted solution of (10) with $J=1 / 4-\sigma$ and $\eta_{\text {meso }}(t, J)$ the solution of (13). We take the initial configurations $n_{\mathrm{ELS}}=1=\eta_{\text {meso }}$. Both solutions, near criticality, behave as

$$
\eta=\left(a_{t} t\right)^{-\alpha} R\left[J\left(a_{t} t\right)^{1 / \nu_{\|}}\right],
$$

with $R$ and $a_{t}$ the corresponding ELS or mesoscopic scaling functions and nonuniversal metric factors. Normalizing $R$ by $R(0)=1$, we can find the metric factors as the amplitude of the power law at the critical point $J=0$. We obtain $a_{t}^{\mathrm{ELS}}=1 / 2$ and $a_{t}^{\text {meso }}=1$ [see Eq. (12)].

To compare $R^{\mathrm{ELS}}$ and $R^{\text {meso }}$ we rescale, for the ELS and the mesoscopic equation, the time by the corresponding metric factors, i.e., $t \rightarrow t / a_{t}$. The solutions are presented in Fig. 1. The data collapse is shown in Fig. 2. It provides a convincing hint that the mesoscopic equation and the ELS model belong to the same universality class. ${ }^{1}$

The differences observed between the two models are consistent with the considered approximations to derive the mesoscopic equation (13). The term "mesoscopic" is justified since it captures the critical behavior of the ELS model for large time and/or small control parameter $J$.

\footnotetext{
${ }^{1}$ The exact solution of (13) for a nonzero initial condition $\eta(0)>0$ gives a small imaginary contribution. Note that we do not observe it numerically, using a standard ordinary differential equation solver. We neglect it as it does not contribute to the singular behavior of the transition. The imaginary part decreases exponentially with time and thus the stationary solution is real since we take $J \geqslant 0$. Moreover, the critical solution $J=0$ is also real $\left[\eta_{\text {meso }}(t, J=0)=\right.$ $1 /(1+t)]$. Finally, since we do not have an exact solution for the ELS equation (10), nothing guarantees that the solutions are also real. However, as for the mesoscopic equation, we do not observe any imaginary parts through numerical solutions.
}

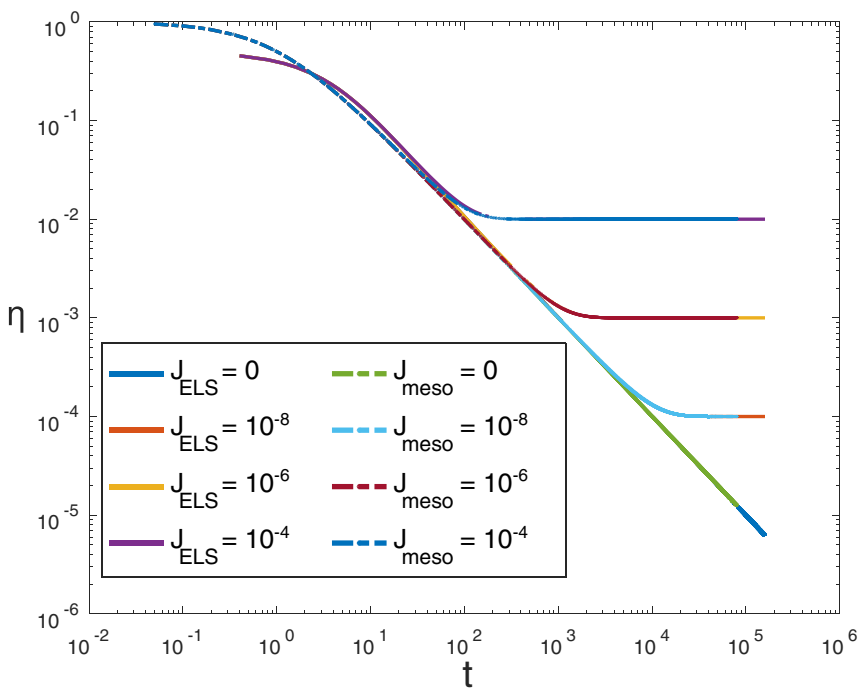

FIG. 1. Solutions of the ELS continuous equation (10) and mesoscopic equation (13) with the time rescaled by $t \rightarrow t / a_{t}$.

\section{FIBER BUNDLE MODEL AS AN ABSORBING PHASE TRANSITION}

In the absence of conservation laws or specific symmetries, it is expected that nonequilibrium phase transitions from an active to a unique absorbing state are described by the directed percolation universality class $[2,6,7]$. Here we show that the mesoscopic ELS behavior, in zero external field, is characterized by a particular symmetry, namely, the particlehole symmetry.

The large-scale behavior of ELS is encompassed by the order parameter mesoscopic equation (13). Rewriting it in term of the density of intact fibers $n$, i.e., $\eta=n-n_{c}\left(n_{c}=1 / 2\right)$, we have

$$
\frac{\partial n}{\partial t}=\lambda n(1-n)-\sigma
$$

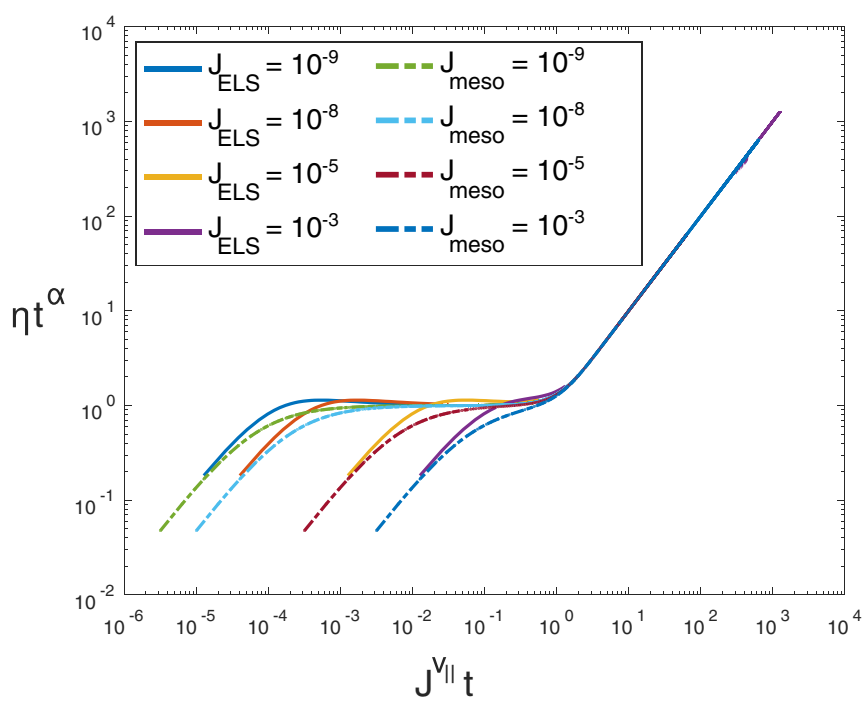

FIG. 2. Data collapse according to the scaling form (16) with $\alpha=1$ and $v_{\|}=1 / 2$. The time is rescaled by $t \rightarrow t / a_{t}$. 
with $\lambda=1$. In zero external field $\sigma=0$, Eq. (17) obeys the particle-hole symmetry, i.e.,

$$
n \leftrightarrow 1-n, \quad \lambda \leftrightarrow-\lambda .
$$

The invariance under this transformation is characterizing the CDP universality class [1,17] (also called compact domain growth). The CDP upper critical dimension is $d_{c}=2$. For $d>$ 2 , the process is exactly described by Eq. (17) with $\sigma=0$. In a CDP process, the dynamics occurs only at boundaries between clusters of active and inactive sites. In other words, using the FBM terminology, the dynamics take place at the domain walls between clusters of intact and broken fibers. Moreover, fibers cannot break (or be created, due to irreversibility) spontaneously; they break only under load redistribution which occurs at the boundaries between clusters of broken and intact fibers.

In this work we focus on ELS and thus we do not have access to spatial features of the FBM. However, the local-load-sharing (LLS) model [10], an FBM for which load redistribution acts locally and whose MF limit is ELS, is precisely characterized by a dynamics that takes place at the interfaces between clusters of intact and broken fibers. Thus, at a phenomenological level, the attempt to relate ELS with CDP is founded. However, in the FBM, unlike in CDP, a broken fiber cannot recover. We expect that it does not impact, on average, the large-scale behavior of the order parameter.

Here we clarify the role played by the external field $\sigma$. The ELS model is driven by $\sigma$, which breaks the particle-hole symmetry. The external field $\sigma$ initiates the primary holes, the seeds, around which load redistribution take place. Indeed, Eq. (3) at step $t=0$ gives $n(1)=1-\sigma$ considering, initially, that all fibers are intact, $n(0)=1$. Then the dynamics occurs first around the seeds and then around the germinative clusters of broken fibers until the system reaches a stable configuration given $\sigma$.

In this section we aimed at linking the FBM with CDP, a well-known process that undergoes an absorbing phase transition. We took advantage of the symmetry of the mesoscopic equation in zero external field to compare formally and phenomenologically ELS with CDP. However, since we work at the MF level we cannot elaborate a unique field theory describing FBM. A mapping of the LLS model to an APT process is needed.

We note that the external field $\sigma$ destroys the absorbing phase and reduces it to a point. Considering $\eta=n-n_{c}$ as the order parameter, the absorbing point is located at $\sigma=\sigma_{c}=$ $1 / 4$. In zero field, CDP has two absorbing states, the empty lattice and the fully occupied lattice reflecting the emerging $\mathbb{Z}_{2}$ symmetry.

\section{EQUAL-LOAD-SHARING AS AN OVERDAMPED LANGEVIN EQUATION}

The ELS fiber bundle model in its dynamical formulation exhibits a dynamical phase transition. It provides, in the vicinity of the critical point, a natural process that ensures a timescale separation between the kinetics of the order parameter and the renaming physical quantities [2]. As we will see, the ELS model can be described through an overdamped
Langevin equation of the form

$$
\frac{\partial \eta(t)}{\partial t}=-\frac{\delta H[\eta]}{\delta \eta}+\zeta(t)
$$

with $H$ the Hamiltonian of the system and $\zeta(t)$ a noise term. Since, in essence, ELS is a mean field, we set $\zeta(t)=0$.

\section{A. Derivation of $\boldsymbol{H}$}

The ELS and other versions of the fiber bundle model are mainly studied in their quasistatic limit. In this picture, fibers are broken one by one. The quasistatic limit corresponds to slowly stretching the fibers. Therefore, it is convenient to introduce the fiber elongation $x=\sigma / n$ since it is assumed that fibers behave as Hookean springs.

The ELS energy contents, defined through the work done on the fiber bundle under increasing load, was recently studied by Pradhan et al. [18]. The total energy content at damage $k=1-n$ and elongation $x$ is

$$
H[x, k]=\theta\left(x^{2}(1-k)+\int_{0}^{k} d \delta\left(P^{-1}(\delta)\right)^{2}\right),
$$

with $\theta=N \kappa / 2, \kappa$ the Hookean constant, and $P^{-1}(\delta)$ the inverse function of the threshold cumulative distribution. The first term of Eq. (19) is the Hookean energy and the second one is the energy dissipated through fiber failures and hence responsible for the formation of holes, that is, clusters of broken fibers.

The equation of state of the system is

$$
0=\frac{\delta H[x, k]}{\delta k}=\theta\left(-x^{2}+\left[P^{-1}(k)\right]^{2}\right)
$$

and thus, since $x$ and $P^{-1}(k)$ are positive quantities,

$$
x=P^{-1}(k)=k,
$$

assuming a uniform threshold distribution on the unit interval. By definition of the elongation we have

$$
x=\frac{\sigma}{n_{*}}=k .
$$

Hence, using $k=1-n_{*}$, we obtain again Eq. (5), the equation of state of ELS. We are interested in catching the stationary critical behavior of the system at the Hamiltonian level in an expression easier to handle than Eq. (19). Since the system equation of state is given by Eq. (5), we can write

$$
\frac{\delta H\left[n_{*}, \sigma\right]}{\delta n_{*}}=n_{*}^{2}-n_{*}+\sigma=0 .
$$

Integrating this expression, we observe that

$$
H[\eta, J]=\eta^{3} / 3-J \eta,
$$

with $\eta=n_{*}-1 / 2$ and $J=\sigma_{c}-\sigma$. We note that this Hamiltonian was previously studied in the context of spinodal nucleation (see Sec. VII).

\section{B. The ELS overdamped Langevin equation}

The ELS critical slowing down (7) provides us with a natural time-length scale separation between the order parameter kinetics and the other physical quantities. These quantities appear as surrounding noise from the order parameter's point 
of view. Therefore, the critical dynamics of ELS may be described by Eq. (18). By inserting the Hamiltonian (24) into this equation, we find the previously introduced mesoscopic equation (13).

This approach is directly inspired by the time-dependent Ginzburg-Landau equation, which is also called model A dynamics [2] describing, for example, the Glauber model. Glauber dynamics is a minimal kinetic extension of the Ising model for which detailed balance ensures that the system relaxes toward the Ising canonical equilibrium probability distribution without conservation of the order parameter. We note that in ELS the order parameter is also not a conserved quantity since fibers break to eventually reach a stable configuration; this justifies Eq. (18). However, unlike model A, ELS is a genuine nonequilibrium process. Thus, there is no detailed balance relation that can be used to derive a noise term for Eq. (18).

\section{NUCLEATION FIELD THEORY AND ELS}

In [12], an analogy between ELS and spinodal nucleation [13] was observed. By studying the mesoscopic behavior of ELS, we show explicitly the underlying reason, which relies on particle-hole invariance of the mesoscopic ELS equation.

The Hamiltonian (24) is formally equal to the field-theoretic description of spinodal nucleation [13]. More explicitly, the Landau-Ginzburg-Wilson Hamiltonian in the mean-field approximation is the free energy

$$
F[\psi]=b \psi^{2}+c \psi^{4}-h \psi,
$$

with $b=a\left(T-T_{c}\right)$ the distance from the critical temperature $T_{c}$, and $a>0$ and $c>0$ two constants. In zero external field $h=0$ and for $T<T_{c}$ (i.e., $b<0$ ), $F$ exhibits the two characteristic symmetric wells. Increasing $h>0$, the well located in negative $\psi$ values becomes shallower and eventually disappears at $h_{s}$ and $\psi_{s}=-(|b| / 6 c)^{1 / 2}$, the spinodal. Then, close to the spinodal, introducing the field $\phi=\psi-\psi_{s}$ and neglecting the irrelevant $\phi^{4}$ term [13], we have

$$
F_{s}[\phi]=\epsilon \phi-\alpha \phi^{3},
$$

with $\epsilon \propto h_{s}-h$ and $\alpha$ a positive constant depending on $b$ and $c$. The mean-field spinodal theory (26) is formally equivalent to Eq. (24).

In Sec. $\mathrm{V}$ we showed that the ELS at the mesoscopic level in zero external field is invariant under the particle-hole symmetry. It allows us to employ the $\mathbb{Z}_{2}$ invariant expression (25) to describe zero-field ELS. Indeed, introducing the latticegas mapping $\psi=2 n-1$, we can readily show that the spinsign symmetry is equivalent to the particle-hole symmetry for the lattice gas

$$
\psi \leftrightarrow-\psi \Longleftrightarrow n \leftrightarrow 1-n .
$$

The lattice-gas variable is suitable to describe ELS since we focus on particle density instead of magnetization.

To conclude, we argued and showed, relying on the particlehole symmetry, why the ELS model can be viewed as a (genuine nonequilibrium) realization of spinodal nucleation.

\section{DISCUSSION}

In this work we derived a mesoscopic equation describing the ELS critical behavior. The mesoscopic equation is invariant under particle-hole symmetry in zero external field. It enables us to formally link ELS with the compact directed percolation model, which describes processes characterized by dynamics occurring at intact broken clusters' boundaries (which is precisely how the FBM dynamics evolves). We described the mesoscopic behavior of ELS as an overdamped Langevin equation. The outcome is the derivation of a Hamiltonian representing the ELS stationary behavior. Due to the particlehole symmetry, we made explicit the formal ELS-spinodal nucleation equivalence.

One of the main outcomes of this work is to link the ELS fiber bundle model, and more generally damage models, to the powerful formalism developed to study nonequilibrium phase transitions. It opens the way for a field-theoretic treatment of such models. In this work we focused on ELS; due to its meanfield nature it is not possible to define a unique field theory for the FBM. Hence, future work should concentrate on spacedependent fiber bundle models such as the local-load-sharing model.

\section{ACKNOWLEDGMENTS}

The authors thank Jonas T. Kjellstadli for interesting discussions. This work was partly supported by the Research Council of Norway through its Centers of Excellence funding scheme, Project No. 262644. M.H. thanks the Swiss National Science Foundation's Early Postdoc. Mobility grant, project No. 171982.
[1] M. Henkel, H. Hinrichsen, and S. Lübeck, Non-Equilibrium Phase Transitions, Vol. 1: Absorbing Phase Transitions (Springer, Berlin, 2008).

[2] U. C. Täuber, Critical Dynamics: A Field Theory Approach to Equilibrium and Non-Equilibrium Scaling Behavior (Cambridge University Press, Cambridge, 2014).

[3] U. Dobramysl, M. Mobilia, M. Pleimling, and U. C. Täuber, Stochastic population dynamics in spatially extended predatorprey systems, J. Phys. A: Math. Theor. 51, 063001 (2018).

[4] J. A. Bonachela and M. A. Muñoz, Confirming and extending the hypothesis of universality in sandpiles, Phys. Rev. E 78, 041102 (2008).
[5] P. Le Doussal and K. J. Wiese, Exact Mapping of the Stochastic Field Theory for Manna Sandpiles to Interfaces in Random Media, Phys. Rev. Lett. 114, 110601 (2015).

[6] H. K. Janssen, On the non-equilibrium phase transition in reaction-diffusion systems with an absorbing stationary state, Z. Phys. B 42, 151 (1981).

[7] P. Grassberger, On phase transitions in Schlögl's second model, Z. Phys. B 47, 365 (1982).

[8] S. Pradhan, A. Hansen, and B. K. Chakrabarti, Failure processes in elastic fiber bundles, Rev. Mod. Phys. 82, 499 (2010).

[9] A. Hansen, P. C. Hemmer, and S. Pradhan, The Fiber Bundle Model (Wiley-VCH, Berlin, 2015). 
[10] S. Sinha, J. T. Kjellstadli, and A. Hansen, Local load-sharing fiber bundle model in higher dimensions, Phys. Rev. E 92, 020401 (2015).

[11] P. Bhattacharyya, S. Pradhan, and B. K. Chakrabarti, Phase transition in fiber bundle models with recursive dynamics, Phys. Rev. E 67, 046122 (2003).

[12] S. Zapperi, P. Ray, H. E. Stanley, and A. Vespignani, First-Order Transition in the Breakdown of Disordered Media, Phys. Rev. Lett. 78, 1408 (1997).

[13] W. Klein and C. Unger, Pseudospinodals, spinodals, and nucleation, Phys. Rev. B 28, 445 (1983).

[14] G. Odor, Universality in Non-Equilibrium Lattice Systems: Theoretical Foundations (World Scientific, Singapore, 2008).
[15] M. Droz and L. Sasvári, Renormalization-group approach to simple reaction-diffusion phenomena, Phys. Rev. E 48, R2343(R) (1993).

[16] P. A. Rey and M. Droz, A renormalization group study of a class of reaction-diffusion models, with particles input, J. Phys. A: Math. Gen. 30, 1101 (1997).

[17] H. K. Janssen, Survival and percolation probabilities in the field theory of growth models, J. Phys.: Condens. Matter 17, S1973 (2005).

[18] S. Pradhan, A. Hansen, and P. Ray, A real space renormalization group procedure for the fiber bundle model, Front. Phys. 6, 65 (2018). 